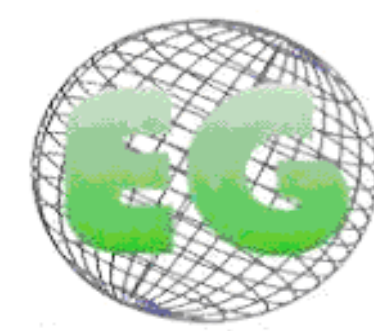

ISSN 1695-6141

$N^{\circ} 23$
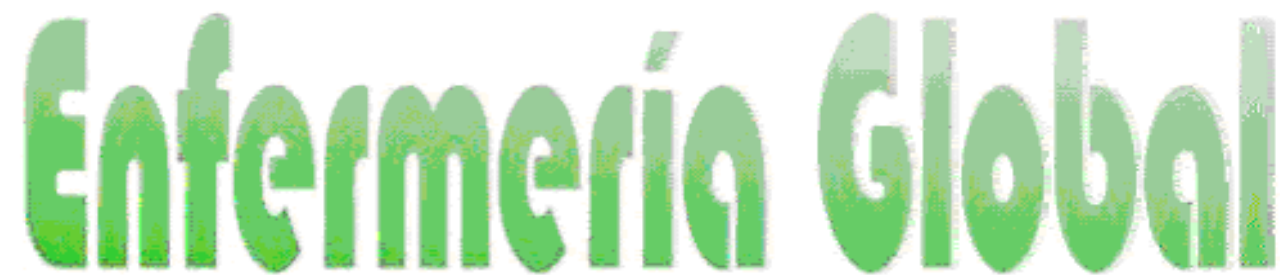

Revista electrónica trimestral de Enfermería

www.um.eslegloball

\title{
Caracterización del ingreso en el hogar en un área de salud del Municipio Marianao
}

Characterization of home admissions in a health area in the Marianao Municipality

\author{
*Fajardo Villarroel, Aurora \\ *Lcda. en Enfermería. Profesora Asistente. Instituto Superior de Ciencias Médicas de La Habana. Cuba. \\ Palabras clave: Ingreso en el hogar; Grupo Básico de trabajo; competencia y desempeño.
}

Keywords: Home admissions; Sanitary work group; Competence and performance.

\section{RESUMEN}

Se estudiaron 400 pacientes del Grupo Básico de Trabajo (GBT) \#2 del Policlínico Docente "Dr. Carlos J. Finlay" del Municipio Marianao, durante el año 2008. El trabajo estuvo encaminado a contribuir al conocimiento de las principales características del ingreso en el hogar, cumpliendo los lineamientos generales y la ética profesional, demostrando que constituye la principal herramienta del binomio médico - enfermera de la familia para garantizar la atención integral al paciente y la familia. Se realizó un estudio descriptivo con carácter retrospectivo enfatizando los resultados en las tablas estadísticas expresadas en números absolutos y relativos con una y dos entradas. Los grupos de edades de mayor incidencia fueron de $40-59$ años con un 38.5\% y más de 60 años con un $24.7 \%$. El sexo femenino predominó en un $65.0 \%$. Los ingresos más frecuentes fueron por hipertensión arterial con un $30.25 \%$, asma bronquial con $24.75 \%$, y las infecciones respiratorias agudas con $12.75 \%$. La mayoría tuvo una estadía mediana con 56.75\%. El $97.75 \%$ de los casos evolucionaron satisfactoriamente lo que demuestra el abnegado trabajo del médico y la enfermera.

\section{ABSTRACT}

We studied 400 patients of the sanitary Work Group \# 2 Polyclinic "Dr. Carlos J. Finlay Marianao Municipality during the year 2008. The work was aimed at contributing to the knowledge of the main characteristics of home admissions by meeting the general guidelines and professional ethics, demonstrating that it constitutes the main tool of the binomial nurse - family doctor to ensure comprehensive care for patients and family. We performed a retrospective descriptive study, emphasizing the results in the statistical tables expressed in absolute and relative numbers with one and two entries. The age groups with the highest incidence was $40-59$ years with $38.5 \%$ and over 60 years with $24.7 \%$. Females predominated in $65.0 \%$. The most frequent admissions were due to high blood pressure with a $30.25 \%$, the $24.75 \%$ for bronchial asthma and acute respiratory infections with $12.75 \%$. The majority $56.75 \%$ had a medium-length stay. $97.75 \%$ of cases recovered satisfactorily, demonstrating the self-sacrificing job of the doctor and nurse. 


\section{INTRODUCCIÓN}

El desarrollo de la Atención Primaria de Salud en Cuba ha sido considerable desde la constitución del Sistema Nacional de Salud. La creación de un médico y enfermera diferente y un nuevo especialista con el fin de alcanzar nuevos niveles de salud, se materializa con el Médico y la Enfermera de la Familia, quienes son los máximos responsables de la salud de los individuos, la familia y la comunidad. ${ }^{(1,2)}$.

Con la creación de los consultorios médicos, el ingreso en el hogar constituye una de las principales acciones médicas a desarrollar, representando un verdadero logro de este Subsistema por todas las ventajas que reporta. El ingreso en el hogar se conceptualiza como la atención médica domiciliaria y continua a pacientes que necesitan de un seguimiento diario de su enfermedad, pero que no conlleve necesariamente internamiento hospitalario, aunque sí encamamiento, aislamiento o reposo ${ }^{(3,4)}$.

La atención hospitalaria a la población constituye una de las formas más importantes de la asistencia médica, no solo por su complejidad, sino por ser la forma más costosa de los tipos de atención. En Cuba este tipo de atención consume el $61.9 \%$ del presupuesto total asignado por el Estado a la salud ${ }^{(4)}$.

El ingreso en el hogar es la principal herramienta del binomio médico-enfermera y profesores del Grupo Básico de Trabajo (GBT) para garantizar la atención integral al paciente y a la familia, logrando una satisfacción plena de la población y verdadera competencia y desempeño de los profesionales. Estas razones y ventajas tanto humanas, profesionales y materiales que proporciona el ingreso domiciliario, nos ha motivado en la realización de este trabajo científico.

\section{OBJETIVOS GENERALES}

1. Contribuir al conocimiento de las principales características del ingreso en el hogar como estrategia fundamental en la Atención Primaria de Salud en Cuba.

\section{OBJETIVOS ESPECÍFICOS}

1. Determinar la distribución de los ingresos por edad y sexo.

2. Identificar las causas más frecuentes de ingresos en el hogar y su estadía.

3. Conocer la evolución de los casos ingresados en el área de salud.

4. Identificar el grado de satisfacción de la población con respecto al ingreso en el hogar.

5. Analizar la situación costo beneficio que reporta el ingreso en el hogar a la Salud Pública y a la economía nacional.

\section{MATERIAL Y MÉTODO}

Se realizó un estudio descriptivo con carácter retrospectivo en el Policlínico Docente "Dr. Carlos J. Finlay" del Municipio Marianao Ciudad de La Habana, Cuba. El universo de estudio fue de 400 pacientes ingresados en el hogar correspondiente al GBT \# 2 de dicho Policlínico durante el año 2008.

Se revisaron las Historias Clínicas individuales y fichas familiares de cada paciente ingresado. Seleccionamos las variables de edad, sexo, causas de ingresos, estadías, evolución de los pacientes y satisfacción. 
Realizamos tablas estadísticas de una y dos entradas, los resultados fueron expresados en frecuencia absoluta y relativa.

En todos los casos se realizaron las respectivas pruebas de hipótesis, siendo el error mínimo permisible $\iota=0.05$.

\section{ANÁLISIS DE LOS RESULTADOS}

Se analizó la distribución de los ingresos en el hogar según las variables de edad y sexo. El mayor número corresponde a los grupos de 40-59 años con 154 casos para un 38.5\% y más de 60 años con 99 casos para un $24 \%$.

Los resultados están dados porque en dichos grupos la prevalencia de afecciones crónicas es más elevada, razón por la cual en nuestro Sistema de Atención Primaria se han creado diferentes programas encaminados a la reducción de la morbi-mortalidad en estas edades.

Predominó el sexo femenino con un número de 260 casos que representa $65.0 \%$ del universo estudiado. Tabla I.

TABLA \# I

Distribución de los ingresos en el hogar según edad y sexo. Grupo Básico de Trabajo \# 2. Policlínico Docente "Dr. Carlos J. Finlay" Año 2008.

\begin{tabular}{|c|c|c|c|c|}
\hline \multirow{2}{*}{ EDAD } & \multicolumn{4}{|c|}{ SEXO } \\
\hline & FEMENINO & $\%$ & MASCULINO & $\%$ \\
\hline$<1$ & 16 & 4.0 & 4 & 1.0 \\
\hline $1-9$ & 23 & 5.75 & 16 & 4.0 \\
\hline $10-19$ & 29 & 7.25 & 14 & 3.5 \\
\hline $20-39$ & 35 & 8.75 & 10 & 2.5 \\
\hline $40-59$ & 99 & 24.75 & 55 & 13.75 \\
\hline$>=60$ & 58 & 14.5 & 41 & 10.25 \\
\hline TOTAL & 260 & 65.0 & 140 & 35.0 \\
\hline
\end{tabular}

FUENTE: Historia Clínica y Fichas Familiares.

Este resultado confirma las estadísticas señaladas por la bibliografía, si tenemos en cuenta que la distribución de los ingresos en el hogar según las causas más frecuentes corresponden a la Hipertensión Arterial , Asma Bronquial e Infecciones Respiratorias Agudas (5). Tabla II. 
TABLA \# II

Distribución de los ingresos en el hogar según

Enfermedades más frecuentes

Grupo Básico de Trabajo \# 2. Policlínico Docente "Dr. Carlos J. Finlay"

Año 2008.

\begin{tabular}{||c||c|c||}
\hline \multicolumn{1}{|c||}{ ENFERMEDADES } & TOTAL & $\%$ \\
\hline \hline Hipertensión Arterial & 121 & 30.25 \\
\hline \hline Asma Bronquial & 99 & 24.75 \\
\hline \hline Enfecciones Respiratorias Aguda & 51 & 12.75 \\
\hline \hline Hepatitis viral & 30 & 7.5 \\
\hline \hline Puerperio & 29 & 7.25 \\
\hline \hline Diabetes Mellitus & 20 & 5.0 \\
\hline \hline Insuficiencia cardiaca y cardiopatía \\
izquémicas.
\end{tabular}

FUENTE: Historias Clínicas y Fichas Familiares.

Podemos apreciar que la literatura plantea que en nuestro país los casos de Hipertensión Arterial son más frecuentes en un $34.83 \%$ en el sexo femenino a partir de los 40 años de edad; de la misma forma en el Asma Bronquial, aunque discreto, el predominio del padecimiento recae en el sexo femenino después de la adolescencia; sin embargo en las infecciones respiratorias hay mayor afectación en el sexo masculino predominando desde la infancia ${ }^{(6)}$.

La distribución de los ingresos en el hogar según las causas más frecuentes por las cuales se practicaban los mismos se muestran en la Tabla II. La mayor incidencia recayó sobre la Hipertensión Arterial descompensada con 121 pacientes para un $30.25 \%$, seguida del Asma Bronquial con 99 pacientes para un 24.75\% y las Infecciones Respiratorias con 51 pacientes para un $12.75 \%$, seguido de otros menos frecuentes. Los resultados aquí obtenidos concuerdan con las principales causa de morbi-mortalidad de nuestro país, además la 
Hipertensión Arterial descompensada desencadena a largo plazo enfermedades vasculares, cerebro-vasculares y cardiovasculares ${ }^{(6)}$.

En cuanto al estadio promedio de los ingresos se aprecia que el mayor número de los pacientes tuvo ingreso de mediana estadía representando el $56.75 \%$ de la población ingresada. Tabla III.

TABLA III

Distribución de los ingresos en el hogar según su estadía

Grupo Básico de Trabajo \#2. Policlínico "Dr. Carlos J. Finlay"

Año 2008.

\begin{tabular}{|c|c|c|}
\hline ESTADÍA & $\overline{\text { PACIENTE }}$ & $\%$ \\
\hline CORTA & 51 & 12.75 \\
\hline$\overline{\text { MEDIANA }}$ & 227 & 56.75 \\
\hline LARGA & 122 & 30.5 \\
\hline$\overline{\text { TOTAL }}$ & 400 & 100 \\
\hline
\end{tabular}

\section{LEYENDA}

$\begin{array}{ll}* \text { CORTA: } & 72 \text { horas } \\ \text { * Mediana: } & \text { hasta una semana } \\ * \text { Larga. } & \text { Más de una semana. }\end{array}$

Esto dependió en gran medida de las afectaciones que presentaron los pacientes. La labor de enfermería estuvo encaminada fundamentalmente al cumplimiento de los tratamientos médicos, vigilancia y estricta observación a los pacientes a través de la visita domiciliaria, así como la realización del proceso de atención de enfermería y la educación sanitaria, enfatizando en la higiene ambiental y personal, apoyo a la esfera emocional a pacientes y familiares entre otras acciones interdependientes e independientes de enfermería. ${ }^{(7)}$.

El $100 \%$ de los familiares afectados con ingresos en el hogar manifestaron plena satisfacción con la atención brindada por el equipo de salud. Esto indica que la política del Sistema de Salud Cubano en cuanto a la Atención Primaria y el ingreso en el hogar marchan satisfactoriamente gracias al cumplimiento de los lineamientos por parte del equipo de salud de la comunidad para el ingreso en el hogar en cada caso que lo requiera.

La evolución de 391 pacientes fue satisfactoria lo que representa un $97.75 \%$, resultado significativo, ya que sólo nueve pacientes requirieron atención hospitalaria para un $2.25 \%$. Tabla IV. 
TABLA IV

Distribución de los ingresos en el hogar según la evolución

Grupo Básico de Trabajo \# 2. Policlínico "Dr. Carlos J. Finlay"

Año 2008.

\begin{tabular}{||c||c||c||}
\hline EVALUACIÓN & NO. & $\%$ \\
\hline \hline SATISFACTORIA & 391 & 97.75 \\
\hline \hline NO SATISFACTORIA & 9 & 2.25 \\
\hline \hline TOTAL & 400 & 100 \\
\hline
\end{tabular}

FUENTE: Historias Clínicas y Fichas Familiares

Pudimos constatar, que el ingreso en el hogar contribuye en gran medida a la adecuada utilización de los recursos hospitalarios; como se analiza en la Tabla $V$ los gastos en Moneda Nacional (MN) que reporta un ingreso en una institución de atención secundaria o terciaria, es superior, lo que provoca que haya que dedicar una proporción considerable de los gastos nacionales a la asistencia de salud en estas instituciones, mientras que la atención comunitaria disminuye los gastos.

Si tenemos en cuenta que en un universo de 51 pacientes, con un ingreso mínimo de tres días en una sala de Medicina, estaríamos ocasionando un gasto de $9302.4 \mathrm{MN}$; ya que el costo por día paciente es de $60.80 \mathrm{MN}$. Por lo tanto con el ingreso en el hogar de 122 pacientes durante 10 días, ahorraríamos $74176.00 \mathrm{MN}$ al estado cubano ${ }^{(8)}$.

En un instituto como el de Cirugía Cardiovascular, un paciente ingresado tiene un costo de 73.79 MN diario. Por lo que 51 pacientes ingresados en el hogar durante tres días, ahorran al país $11289.87 \mathrm{MN}$.

TABLA V

Análisis estadístico del costo de ingreso en un Hospital Referencia Nacional

Año 2008.

\begin{tabular}{||c|c||}
\hline ESPECIALIDAD & COSTO POR DÍA (MN) \\
\hline \hline CARDIOLOGÍA & 73.79 \\
\hline \hline MEDICINA & 60.80 \\
\hline
\end{tabular}

FUENTE: Anuario Estadístico. Año 2008. 
Representa también una ventaja para los pacientes ya que transcurre su enfermedad en un entorno familiar, recibiendo apoyo del equipo de salud, la familia y la comunidad.

En cuanto a los principios éticos pudimos constatar que el ingreso en el hogar tenía un carácter completamente voluntario, no debiéndose realizar el mismo si existe duda o rechazo, cumpliendo así con el principio de autonomía y el consentimiento informado. Así mismo cuando los especialistas básicos, clínicos pediatras y ginecobstetras controlan periódicamente las visitas a los consultorios de las zonas urbanas, los ingresos en el hogar, se aplica la beneficencia y no la maleficencia, de la misma forma que cuando se plantea que los ingresos en el hogar deben ser visitados al menos diariamente por el médico y la enfermera de la familia que es la encargada de practicar en el hogar las acciones y procederes de enfermería proporcionándole al paciente bienestar y pronta recuperación.

\section{CONCLUSIONES.}

1. Las edades de mayor incidencias o de ingreso en el hogar fueron de 40-59 años con un $38.5 \%$ y más de 60 años con un $24.7 \%$. El sexo femenino predominó con un $65 \%$ sobre el masculino.

2. Las causas que con mayor frecuencia requirieron ingreso domiciliario, fueron la Hipertensión Arterial, 30.25\%, seguida del Asma Bronquial, 24.75\% y luego las infecciones respiratorias agudas, $12.75 \%$.

3. La estadía en el hogar predominante fue la mediana para un $56.75 \%$

4. El $97.75 \%$ de los ingresados evolucionó satisfactoriamente.

5. El grado de satisfacción de la población en cuanto al ingreso en el hogar fue elevado, demostrando así que el desempeño de los profesionales del equipo de salud fue óptimo, respetando siempre los principios de la ética.

6. El ingreso en el hogar reporta una mejor utilización de los recursos hospitalarios así como un adecuado aprovechamiento del producto nacional bruto destinado a la Salud Pública.

\section{RECOMENDACIONES.}

Se impone la necesidad de su perfeccionamiento y aplicación al máximo de potencialidades ya que el ingreso en el hogar ha impactado en el uso y ahorro de la cama hospitalaria, produciendo un efecto económico positivo para nuestro país.

\section{REFERENCIAS BIBLIOGRÁFICAS}

1. Álvarez Sintes, R: Temas de Medicina General Integral. La Habana; Ed. Ciencias Medicas ,2001.

2. Sánchez Santos, Leonardo: introducción de la medicina integral. Ciudad de la Habana, Ed. Ciencias medicas ,2001.

3. República de Cuba. MINSAP. Carpeta Metodológica 2001.

4. Lectura de Filosofía, Salud y Sociedad. La Habana.Ed. Ciencias Médicas, 2000. 
5. Viña Pérez O, López Montaño JL, Gafas González C, Duménigo Arias O. Ingreso domiciliario en un consultorio del médico de la familia. Rev Electrónica Archivo Médico de Camagüey. 2004; 6(3).

6. Enfermería Familiar y Socia: Colectivo de Autores. En: Atención Primaria De Salud. Cap 1, Ed Ciencias Medicas, 2004.

7. Programa de Especialización en Enfermería Comunitaria. La Habana. ECIMED. 2005.

8. Dirección Nacional de Estadísticas. Anuario Estadístico 2007. MINSAP. 